\title{
PHYSICS CONTRIBUTION
}

\section{IMRT FOR IMAGE-GUIDED SINGLE VOCAL CORD IRRADIATION}

\author{
Sarah O. S. Osman, M.Sc., Eleftheria Astreinidou, Ph.D., Hans C. J. de Boer, Ph.D., \\ Fatma Keskin-Cambay, M.D., Sebastiaan Breedveld, M.Sc., Peter Voet, R.T.T., \\ Abrahim Al-Mamgani, M.D., Ben J. M. Heijmen, Ph.D., and Peter C. Levendag, M.D., Ph.D. \\ Department of Radiation Oncology, Erasmus Medical Center-Daniel den Hoed Cancer Center, Rotterdam, The Netherlands
}

\begin{abstract}
Purpose: We have been developing an image-guided single vocal cord irradiation technique to treat patients with stage T1a glottic carcinoma. In the present study, we compared the dose coverage to the affected vocal cord and the dose delivered to the organs at risk using conventional, intensity-modulated radiotherapy (IMRT) coplanar, and IMRT non-coplanar techniques.

Methods and Materials: For 10 patients, conventional treatment plans using two laterally opposed wedged 6-MV photon beams were calculated in XiO (Elekta-CMS treatment planning system). An in-house IMRT/beam angle optimization algorithm was used to obtain the coplanar and non-coplanar optimized beam angles. Using these angles, the IMRT plans were generated in Monaco (IMRT treatment planning system, Elekta-CMS) with the implemented Monte Carlo dose calculation algorithm. The organs at risk included the contralateral vocal cord, arytenoids, swallowing muscles, carotid arteries, and spinal cord. The prescription dose was $66 \mathrm{~Gy}$ in 33 fractions. Results: For the conventional plans and coplanar and non-coplanar IMRT plans, the population-averaged mean

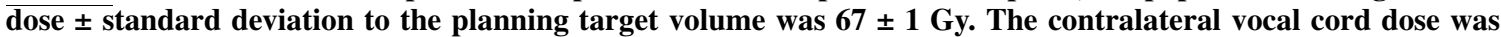
reduced from $66 \pm 1 \mathrm{~Gy}$ in the conventional plans to $39 \pm 8 \mathrm{~Gy}$ and $36 \pm 6 \mathrm{~Gy}$ in the coplanar and non-coplanar IMRT plans, respectively. IMRT consistently reduced the doses to the other organs at risk.

Conclusions: Single vocal cord irradiation with IMRT resulted in good target coverage and provided significant sparing of the critical structures. This has the potential to improve the quality-of-life outcomes after RT and maintain the same local control rates.
\end{abstract}

(C) 2012 Elsevier Inc. Open access under the Elsevier OA license.

Image-guided radiotherapy, Intensity-modulated radiotherapy, Early glottic carcinoma, Normal tissue sparing, Quality of life.

\section{INTRODUCTION}

The ultimate goal of radiotherapy (RT) is the total eradication of tumors while minimizing the toxicity to the surrounding healthy structures. In early glottic carcinoma treated with RT, large box fields, using wedged parallel opposed photon beams, have been used conventionally. The tumor-free contralateral vocal cord, arytenoids, thyroid cartilage, and all muscles responsible for opening and closing the vocal cords receive the full dose of $66 \mathrm{~Gy}$. In this conventional method, the swallowing muscles, carotid arteries, thyroid gland, and thyroid and cricoid cartilages among other structures, are partly in field. Exposure of these structures to high radiation doses (fully or partially) could lead to an increased probability of complications that negatively influence the quality of life of these patients. Typical complications have involved voice/ speech impairment, diet problems (swallowing, trismus), arytenoid edema, an increased risk of strokes, and reduced treatment options for previously irradiated patients (1-3).

Very good local control rates have been obtained with simple parallel opposed beams used conventionally. However, efforts should be made to reduce the dose received by the tumor-free laryngeal structures to reduce the radiationrelated complications in this group of patients. Reducing the dose received by the organs at risk (OARs) might also help in increasing the treatment options for patients with second primary tumors in the vicinity of the treated tumor. In patients with successfully treated Stage T1a glottic cancer, the incidence of the development of a second primary tumor (commonly in the upper aerodigestive track) at 5, 10, and 15 years was reported to be $23 \%, 44 \%$, and $48.7 \%$, respectively (4). Given the large radiation treatment fields presently used
Reprint requests to: Sarah O. S. Osman, M.Sc., Department of Radiation Oncology, Erasmus Medical Center-Daniel den Hoed Cancer Center, Groene Hilledijk 301, P.O. Box 5201, Rotterdam 3008 AE The Netherlands. Tel: (+31) 1-0-704-2692; Fax: (+31) 10-7041012; E-mail: s.osman@erasmusmc.nl
Presented, in part, as a poster at the 10th Biennial European Society for Therapeutic Radiology and Oncology Meeting, Maastricht, The Netherlands, 2009.

Funded in part by Elekta Oncology Systems.

Conflict of interest: none.

Received April 29, 2010, and in revised form Dec 7, 2010. Accepted for publication Dec 8, 2010. 

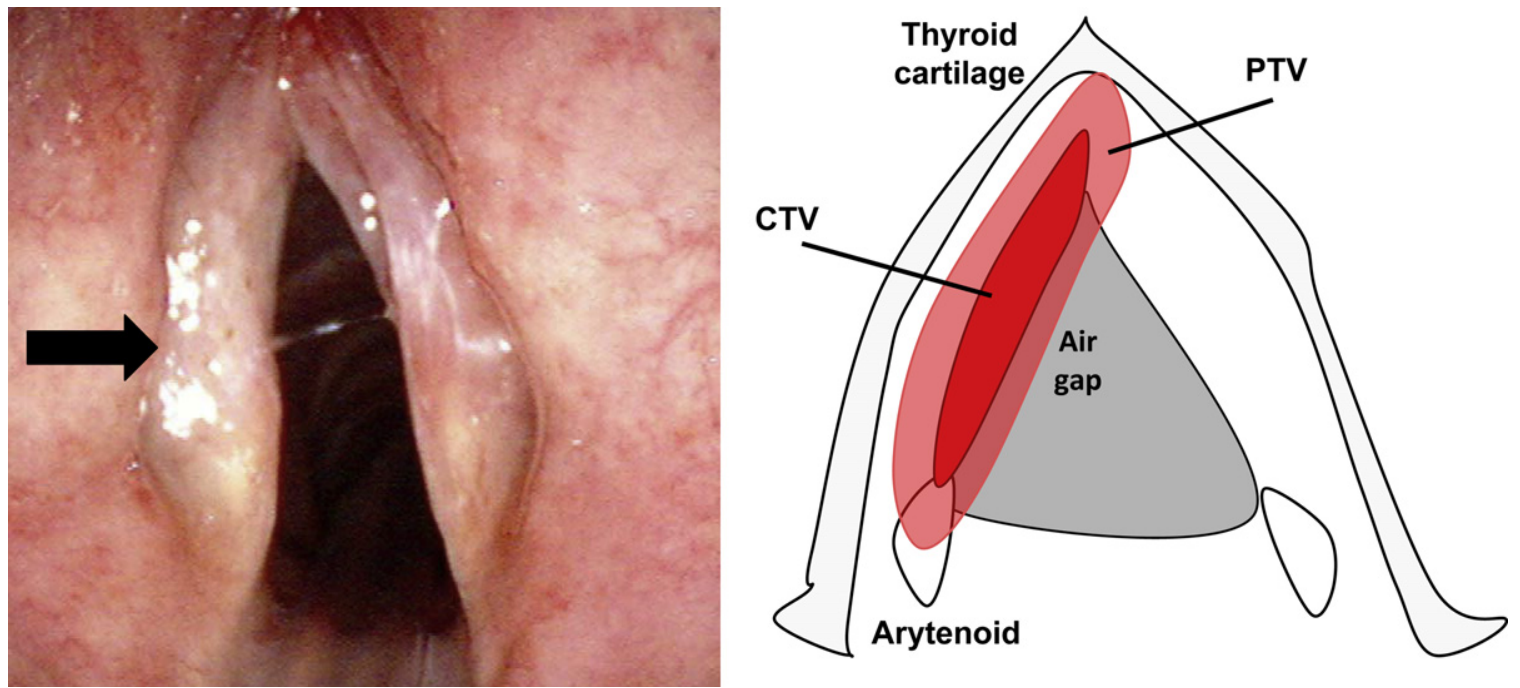

Fig. 1. (Left) Endoscopic photograph of tumor on one vocal cord and (Right) schematic drawing showing delineations of clinical target volume (CTV) and corresponding planning target volume (PTV).

for these patients, those second primary tumors have usually been treated surgically with generous surgical margins that significantly deteriorate the patients' quality of life after treatment (5).

At the Erasmus Medical Center, we have been developing a new focused irradiation technique for early glottic cancer. The introduction of a new technique brings with it the responsibility of investigating, testing, and verifying all aspects of the RT process. The geometric uncertainties must be reduced and the residual uncertainties accounted for by ensuring adequate safety margins for treatment planning. Accurate dose calculation and treatment delivery are also crucial.

In a previous study, we investigated and quantified the intrafraction respiratory motion of the vocal cords using fourdimensional computed tomography (CT) scans. It was found that the movement of the vocal cords with breathing is minimal (6). In another study, it was shown that it is possible to reduce the interfraction setup errors to submillimeter values using cone beam $\mathrm{CT}$ scans in an on-line correction protocol (7).

In the present study, we compared the tumor dose coverage and dose delivered to the OARs using conventional parallel opposed fields with dose delivery using computeroptimized coplanar and non-coplanar beam setups and intensity-modulated beam profiles. The dose calculations were performed using a Monte Carlo (MC)-based treatment planning system (TPS).

\section{METHODS AND MATERIALS}

\section{Patients, planning CT scans, and delineations}

Ten patients who had been conventionally treated for early-stage glottic carcinoma (Stage T1aNOM0) were included in the present study. All patients had undergone four-dimensional CT scanning (Somatom Sensation Open, Siemens Medical Solutions, New York, NY) before treatment, reconstructed in eight breathing phases. The CT resolution was $1 \times 1 \times 1 \mathrm{~mm}^{3}$. For each patient, the reconstruction representing the local anatomy in its average respiratory position was used for planning. The OARs contoured were the contralateral (CL) vocal cord, arytenoids, swallowing muscle at the level of the vocal cords (inferior constrictor muscles [ICM], ipsilateral and CL strap muscles), carotid arteries, thyroid and cricoid cartilages, and spinal cord. The craniocaudal extensions of the contours for these OARs were the $\mathrm{C} 2$ and $\mathrm{C} 6$ vertebrae.

In Fig. 1, an example of a Stage T1 tumor confined to one vocal cord is shown, as well as a schematic drawing of the delineation method used. The clinical target volume (CTV) was defined as the whole affected vocal cord as a conservative measure.

\section{Margins}

In line with the International Commission on Radiation Units and Measurements recommendations, a planning target volume (PTV) was constructed around the CTV. A standard margin formula: $M=2.5 \Sigma_{t o t}+1.64 \sigma_{t o t}-1.64 \sigma_{p}$, where $\sum_{t o t}=$ $\sqrt{\sum_{\text {intrafraction }}^{2}+\sum_{\text {interfraction }}^{2}}, \quad \sigma_{\text {tot }}=\sqrt{\sigma_{\text {intrafraction }}^{2}+\sigma_{\text {intrafraction }}^{2}+\sigma_{p}^{2}}$ and $\sigma_{p}$ is the standard deviation describing the penumbra width, was used (8). The mean penumbra width was $0.4 \mathrm{~cm}$ (the smallest distance between the $95 \%$ and $50 \%$ isodose lines in the plans). Using the CT scan with the tumor in its average position for planning, the systematic respiratory error could be reduced to nearly 0 , and the respiratory motion contribution to the margins stemmed from purely random errors. We included the uncertainties from the intrafraction respiration (6), and the residual interfraction setup errors (assuming on-line setup corrections to be performed), as measured and explained in our previously published study (7). For prolonged treatment sessions ( $\geq 15 \mathrm{~min}$ ), the effects of the intrafraction setup errors might not be negligible and should be accounted for (9). Nevertheless, it has generally been agreed that the magnitude of the intrafraction setup errors will be less than that of the interfractional setup errors (10). In the present study, the intrafraction setup uncertainties were estimated to be equal to the interfraction uncertainties as a reasonable (upper limit) estimate. Using this procedure, margins of $0.5,1.7$, and $0.8 \mathrm{~mm}$ in the mediolateral, craniocaudal, and anteroposterior directions were calculated. Given the $1 \mathrm{~mm}^{3}$ 
Table 1. Wish list used in iCycle showing constraints and objectives used in optimization

\begin{tabular}{|c|c|c|c|}
\hline & \multicolumn{3}{|c|}{ Constraint } \\
\hline & Volume & Type & Limit (Gy) \\
\hline & PTV & Minimum & 63 \\
\hline & PTV & Maximum & 68 \\
\hline & Spinal cord & Maximum & 45 \\
\hline & Unspecified tissue & Maximum & 70 \\
\hline & \multicolumn{3}{|c|}{ Objective } \\
\hline Priority & Volume & Type & Goal (Gy) \\
\hline 1 & CL vocal cord & Mean & 30 \\
\hline 2 & CL arytenoid & Mean & 50 \\
\hline 3 & Ipsilateral arytenoid & Mean & 66 \\
\hline 4 & ICM & Mean & 26 \\
\hline 5 & Unspecified tissue & Minimal EUD & 10 \\
\hline
\end{tabular}

Abbreviations: PTV = planning target volume; $\mathrm{CL}=$ contralateral; ICM = inferior constrictor muscles; EUD = equivalent uniform dose.

resolution of the CT scan, practical margins of $2 \mathrm{~mm}$ in threedimensions were used for the present study.

\section{Treatment plans}

Conventional plans. Conventional clinical treatment plans using two laterally opposed wedged photon beams were performed using the XiO TPS (version 4.33, Elekta-CMS). The superior border of the treatment fields was the hyoid bone, and the inferior border was the lower edge of the cricoid cartilage. The fields were $\sim 1$ $\mathrm{cm}$ "falling off" anteriorly, and the posterior border was at the anterior vertebrae (11). The prescribed dose to the "box" outlined by the treatment fields was $66 \mathrm{~Gy}$ at the $100 \%$ isodose, given in 33 fractions at six fractions weekly. The dose was calculated using the fast superposition algorithm in $\mathrm{XiO}$.

Design of IMRT plans and optimization procedure. An inhouse-developed IMRT/beam angle optimization algorithm (iCycle) was used to obtain the beam angles for each patient using a set of standardized constraints and objectives (12). iCycle uses a list with constraints and prioritized objectives (a "wish list") for beam angle optimization (Table 1). The constraints were hard and must be fulfilled at all times. The objectives were processed in priority. The highest prioritzed objective was to minimize the mean dose to the CL vocal cord to an acceptable level of $30 \mathrm{~Gy}$ (i.e., its "goal"). The second priority was to minimize the mean dose to the CL arytenoid to an acceptable level of $50 \mathrm{~Gy}$, and so forth for the ipsilateral arytenoid, ICM, and unspecified tissue. The list was processed in 2 phases. In the first phase, it sought to attain the goals as well as possible. In the second phase, the objectives for which the goal could be attained were minimized to their fullest. From this wish list, iCycle sequentially adds beams to the plan to a maximum of five beams.

The optimized iCycle beam angles were then imported into the Monaco TPS, version 1.00 (Elekta-CMS) to generate plans with a MC dose calculation algorithm. In the Monaco TPS, the plans were configured using biological and physical constraints (1315). For the PTV, a Poisson cell kill model was used combined with a quadratic overdose constraint to avoid hot spots inside the PTV. For the CL vocal cord and the spinal cord, serial complication models were used to limit the maximal dose to both structures. Quadratic overdose constraints were also applied to limit the dose to unspecified tissue. The beams were then segmented, and their weights were optimized in the second optimization phase using a photon MC dose engine (XVMC) based on the voxel MC code (16).

All calculations for the IMRT plans were done for 6-MV photons with a calculation grid spacing of $1.5 \mathrm{~mm}$. The accelerator used was an Elekta Synergy with 40 multileaf collimator leaf pairs, and the leaf width at the isocenter was $4 \mathrm{~mm}$ (Elekta Beam Modulator, Elekta Oncology Systems, Crawley, UK). The minimal segment size allowed was $0.5 \mathrm{~cm}^{2}$. The isocenter was set to the center of the CTV. The dose prescribed to the PTV was $66 \mathrm{~Gy}$, given in 33 fractions (six fractions weekly) using the clinical fractionation scheme. The PTV partially consist of air. No dose was deposited in that part of the PTV. The Monaco TPS feature "auto flash" creates a flash margin of the voxels that extends beyond the surface of the patient (into the air cavity in this case) so that the prescribed dose can be achieved. A "flash margin" of $2 \mathrm{~mm}$ around the PTV was used in planning. Thus, dose coverage was also provided for the moving vocal cord (CTV) in and out of the air gap during breathing and in the case of setup errors.

Plan evaluation. The population-averaged dose-volume histograms (DVH), mean doses, and, in some cases, maximal doses were used to evaluate and compare the different plans. For the CTV and selected OARs, the generalized equivalent uniform doses [EUD(a)] (17) were calculated and compared for the different planning techniques. The values of the tissue-specific parameter $a$ used for the CTV were $a=-5$ and $a=-20$, because no clear data are available about the values that should be used for these tumors. For the OAR, $a=2.2$ was used, as recently recommended by Rancati et al. (2). Statistical analysis was performed in MatLab using pairwise Wilcoxon signed-rank tests at a significance level of $p$ $\leq .05$. Separate analyses were performed to compare conventional planning with the IMRT coplanar plans and to compare the IMRT coplanar and IMRT non-coplanar plans; no multiple comparison was performed. The fractional volumes of the PTV and CTV that received $\geq 95 \%$ of the prescription dose were also reported. For the OARs, the population-averaged mean doses ( \pm standard deviation) and the maximal doses to the spinal cord and carotid arteries were reported. It has been recommended to keep the dose in the larynx less than $\sim 66$ Gy for vocal function preservation (2). Therefore, the volume of the CL vocal cord that received $\geq 95 \%$ of the prescribed dose or more was also reported. It has also been recommended to limit the mean dose to the non-involved larynx to $40 \mathrm{~Gy}$, because the published data have indicated threshold doses of 40-45 Gy for the onset of other functional complaints $(2,18)$. Therefore, we have also reported the fraction of the volume of the CL vocal cord that received a dose of 40 Gy. Furthermore, the volume of the carotid arteries that received $\geq 35$ Gy are also reported, because reports have been published of complications with carotid doses 35 Gy (3).

\section{RESULTS}

\section{iCycle optimized coplanar and non-coplanar beam angles}

For each patient, a coplanar and non-coplanar set of optimized beam angles was obtained. The distribution of the iCycle beam angles for the 10 coplanar plans is shown in Fig. 2 for tumors on the left vocal cord. Generally, anterior beams have been favored with a wide range of angles. The class solution beam angles used were indicated with 


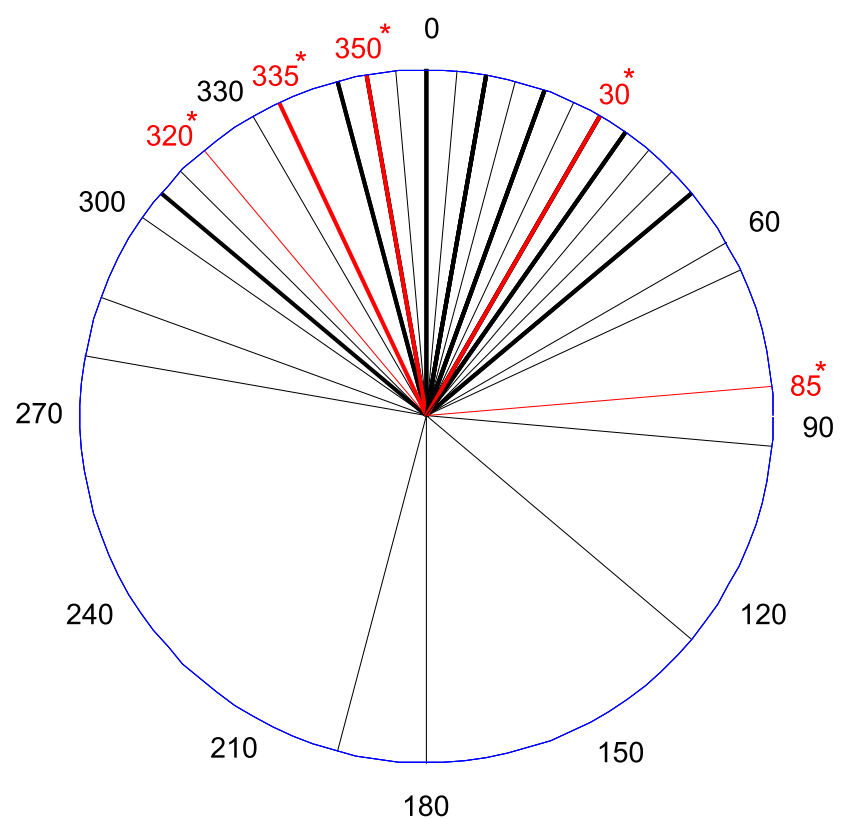

Fig. 2. iCycle coplanar beam distributions for left vocal cord tumor. Optimized angles for right side tumors have been mirrored for this representation. Thicker lines indicate directions chosen more than once. Class solution angles shown in red with asterisk.

asterisks. Non-coplanar iCycle optimized beam angles are presented in Table 2.

\section{Conventional technique vs. coplanar and non-coplanar IMRT}

The beam setups obtained with iCycle were used to plan the treatments in the Monaco TPS. Because of the observed similarity in the selected beam angles for the patients (Fig. 2), we selected one set of five beam angles and tested whether this could be used as a class solution for all patients. This could serve for the simplicity and standardization of treatment delivery in practice. For patients with tumor on the left vocal cord, beam angles of $30^{\circ}, 85^{\circ}, 320^{\circ}, 335^{\circ}$, and $350^{\circ}$ were used. For those with right vocal cord tumors, mirrored angles were used. Figure 3 shows the comparisons between doses received by the different structures, using the individualized beam arrangement and the class solution. No significant differences in the delivered doses to the different structures were observed (all $p>0.05$ ). This could have been a result of the degeneracy of the solution space $(12,19)$. Although not significant, the class solution seemed to result in somewhat better sparing for some OARs. This could have resulted from the different dose calculation algorithms used; the pencil beam algorithm without adequate MLC segmentation in iCycle in which optimized IMRT beam angles were generated, and in Monaco, with a MC-based dose engine and full MLC segmentation. For the sake of simplicity, all the following results for coplanar IMRT plans were produced with the proposed class solution. For non-coplanar plans, the individualized beam angles, as obtained using iCycle (Table 2), were planned in Monaco, because it was more complicated to cluster the beam and couch angles.

Figure 4 shows the dosimetric comparisons among the conventional, coplanar IMRT, and non-coplanar IMRT plans. Comparing the conventional plans with the IMRT coplanar plans, similar mean doses to the CTV $(p=0.2)$ and PTV $(p=0.1)$ were obtained with both techniques. Furthermore, almost all OARs (CL vocal cord, CL arytenoid, carotid arteries, ICM, thyroid, cricoid, and strap muscles) were significantly spared with the IMRT plans compared with the conventional plans (all $p=0.002$, except for the ipsilateral carotid arteries $p=0.004)$. The PTV extended to partially cover the ipsilateral arytenoids. Accordingly, doses similar to the conventionally delivered doses were achieved using IMRT $(p=0.4)$. Compared with coplanar IMRT, only the $\mathrm{CL}$ arytenoid was spared more when using non-coplanar beams $(p=0.01)$.

For coplanar IMRT plans, the smallest field size was $2 \times 2.25 \mathrm{~cm}^{2}$, and each plan had on average four segments/ beam. The average number of monitor units required to deliver the dose was $441 \pm 81$. Similarly, for non-coplanar plans, an average of four segments/beam was used, and the smallest field size was $2 \times 2 \mathrm{~cm}^{2}$. The average number of monitor units was $509 \pm 145$.

In Fig. 5, a typical example of the dose distributions of a conventional plan and IMRT coplanar and non-coplanar setups are shown for 1 patient. The superiority of single vocal cord irradiation (SVCI) is clearly visible. This was also evident in Fig. 6, in which the population mean DVHs for the different planning techniques are presented.

For the CTV, CL vocal cord, and the arytenoids, the EUD mean values ( \pm standard deviation) obtained from the different planning techniques are presented in Table 3. The data show substantial and statistically significant sparing of the CL vocal cord and CL arytenoid for SCVI with IMRT. The $\operatorname{EUD}(-5)$ and $\operatorname{EUD}(-20)$ for the CTV were slightly (but

Table 2. iCycle individualized non-coplanar beam arrangements

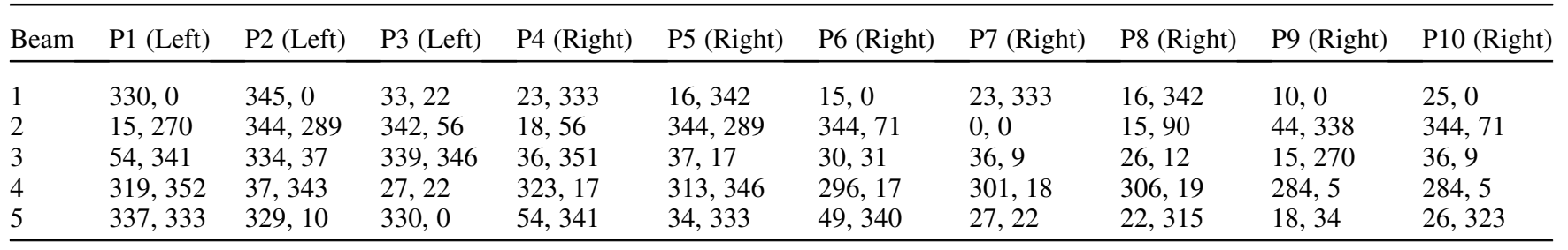




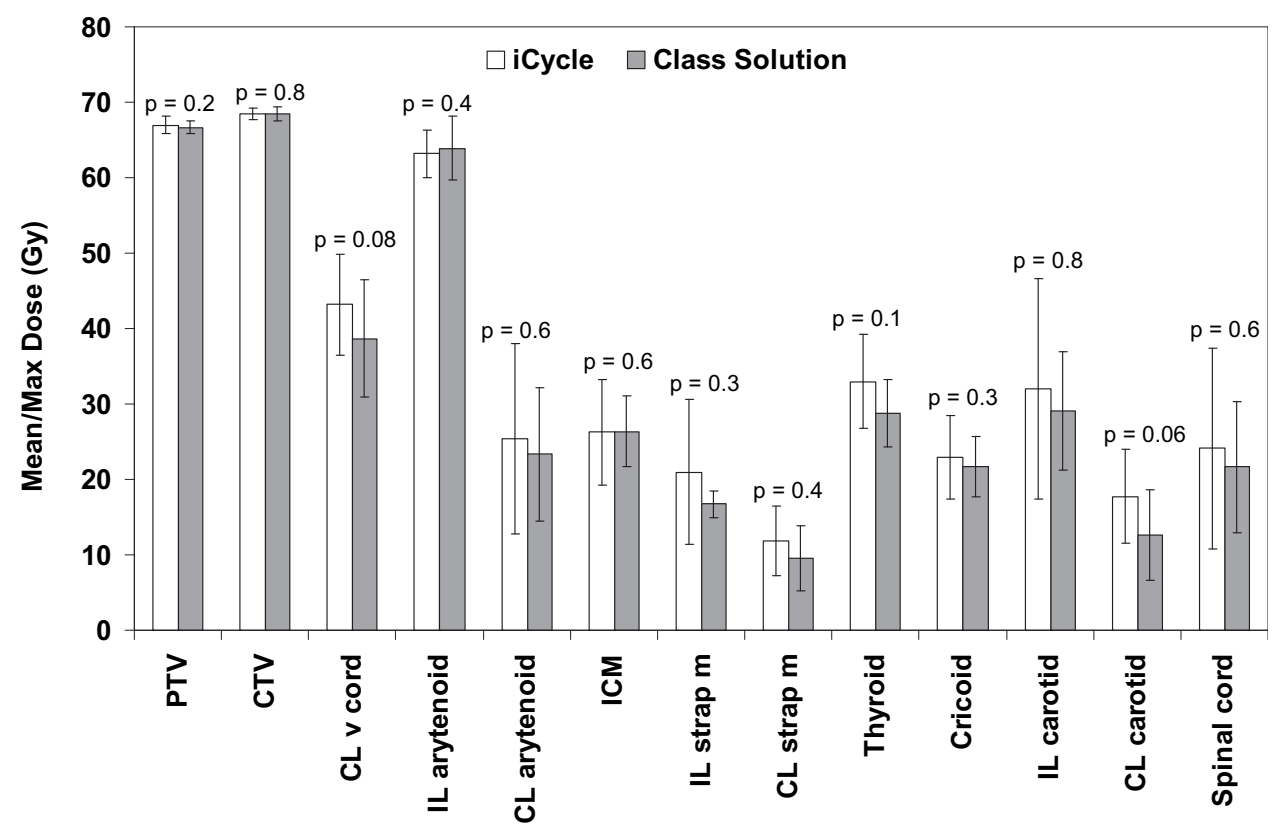

Fig. 3. Mean/maximal doses to different structures from coplanar individualized (iCycle) beam angles and for class solution. For carotid arteries and spinal cord, maximal doses shown. Error bars represent \pm standard deviation. $p$ Values shown for Wilcoxon signed-ranked tests for individualized beam setups vs. class solution doses. CL = contralateral; $\mathrm{v}=$ vocal $; \mathrm{IL}=$ ipsilateral $; \mathrm{m}=$ muscle.

statistically significantly) elevated in the IMRT plans compared with the conventional plans, showing that the large reduction in OAR doses with IMRT could be obtained while also giving a slightly greater dose to the CTV. Several dose-volume parameters for the CTVs and the contralateral vocal cord are listed in Table 4. The percentage of the CTV receiving $\geq 95 \%$ of the dose was, for all plans, $\geq 99 \%$. Only $86 \%$ and $88 \%$ of the PTV received $\geq 95 \%$ of the prescribed dose in the coplanar and non-coplanar plans (see PTV DVH in Fig. 6). This is due to the fact that the PTV extends into the air gap of the larynx. Applying a flash margin (see the "Methods and Materials" section), similar fluences were deposited to the PTV (i.e., opening the leaves to conform to a virtual target). The whole CL vocal cord received $\geq 95 \%$ of the prescribed dose in the current conventional plans; however, in the IMRT plans, only a small fractional volume received such high doses.

On average, the percentage of the volume of the carotid arteries that received a dose of $\geq 35$ Gy in the clinical plans was $86 \%$ for the ipsilateral carotid and $92 \%$ for the CL carotid artery. However, a major reduction in the dose received to both carotid arteries was achieved using SVC IMRT because no parts of the arteries received any dose $>35$ Gy (see also the maximal dose to the carotid arteries in Fig. 4 and the DVHs in Fig. 6).

\section{DISCUSSION}

We have conducted a planning study for one-sided early glottic cancer to compare the conventional RT technique (using wedged parallel opposed fields) with highly focused IMRT. Although IMRT might not be necessary to control early-stage squamous cell carcinoma of the larynx, the added value of normal tissue sparing has made it clinically relevant. We found the dose received by healthy structures in the vicinity of targets can be significantly reduced with the proposed technique without compromising the target coverage (Tables 3 and 4 and Fig. 4).

In the present report, we have proposed a class solution for coplanar beam angles to be used as a template for SVCI in the treatment of early-stage glottic cancer. This class solution was derived from full IMRT/beam angle optimization for the patients in the present study using an in-house-developed optimizer. Comparing the dose parameters for the different OARs using the individualized plans with respect to the proposed class solution, little difference was found, suggesting that the class solution is a valid template (Fig. 3). Using this class solution will reduce the complexity and allow quicker plan generation compared with individualized beam setups. Adding non-coplanar beams resulted in little improvement in plan quality (Fig. 4). Having non-coplanar beams would significantly increase the treatment time compared with coplanar treatment. Moreover, it will introduce positioning difficulties that might not be justified by the small gain in OAR dose reduction.

As demonstrated in Fig. 4 and Table 3, SVCI results in major sparing of the CL vocal cord, CL arytenoid, ICM, strap muscles, thyroid and cricoid cartilages, and carotid arteries compared with conventional RT. For the ipsilateral arytenoid and anterior commissure, we allowed high doses (similar to that with the conventional plans) to avoid target underdosage. For the IMRT plans, the dose to the spinal cord increased with respect to the conventional plans but remained far below the specified limit of $45 \mathrm{~Gy}$. 


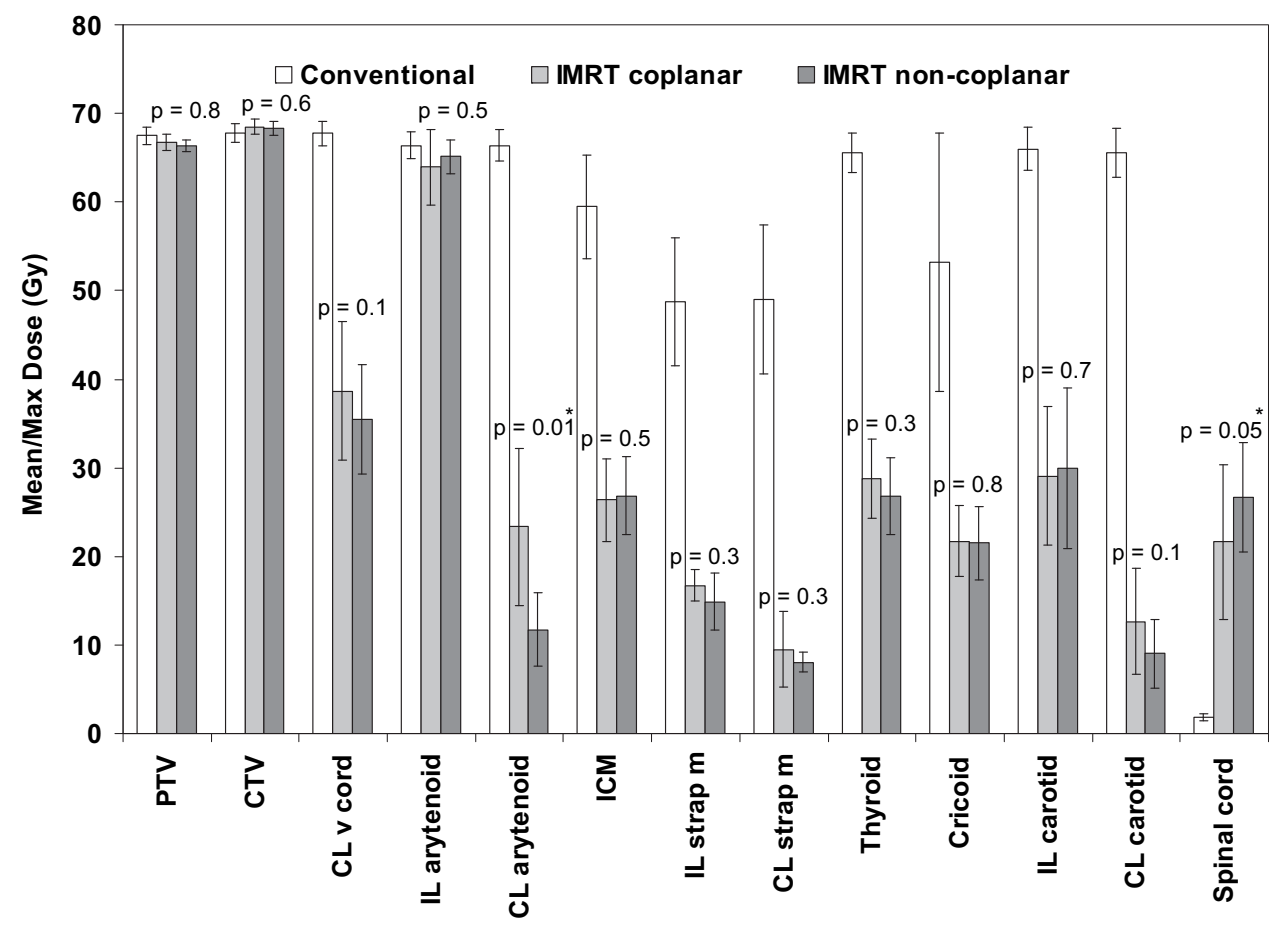

Fig. 4. Delivered doses for planning target volume (PTV) and organs at risk (OARs) from conventional and intensitymodulated radiotherapy (IMRT) coplanar and non-coplanar plans. For carotid arteries and spinal cord, population average maximal doses shown. For other structures, mean doses depicted. Error bars represent \pm standard deviation. $p$ Values shown for Wilcoxon signed-ranked tests for doses delivered using IMRT coplanar vs. non-coplanar beam setups. CL = contralateral; $\mathrm{v}=$ vocal; $\mathrm{IL}=$ ipsilateral; $\mathrm{m}=$ muscle. ${ }^{*}$ Statistically significant.

As mentioned in the "Methods and Materials" section, analysis of the target coverage using the PTV concept would be inaccurate for SVCI because of the air present in the volume. The use of the flash margin should guarantee proper target dose delivery in the case of motion. This is a topic for additional investigation.

Although RT was reported superior to laser surgery in terms of voice preservation, a number of studies have reported that the voice does not return to normal after RT $(20,21)$. Other studies comparing laser surgery and RT as treatment options reported comparable local control and voice quality outcomes from the two treatment modalities (22). These stud- ies compared RT techniques using conventional "box" RT fields (with high doses to all structures included) with localized laser surgery. This provided a strong motive to hypothesize that more focused RT techniques could provide better results in terms of quality of life compared with laser surgery. It could also provide better local control among this group of patients by offering the potential for dose escalation. The increased sparing of the OARs might also allow the application of hypofractionation schemes to reduce the overall treatment time and increase patient comfort.

It has generally been agreed that reducing the doses to the laryngeal structures has the potential to reduce the

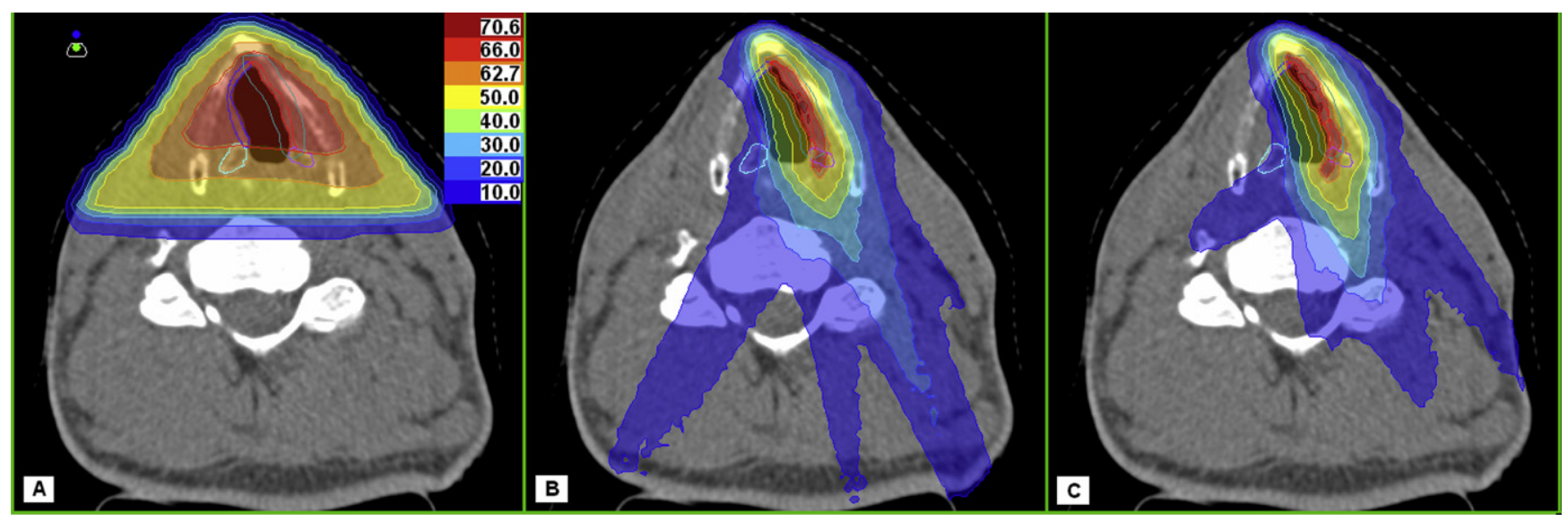

Fig. 5. Transverse views at isocenter level for 1 patient showing dose distributions for (A) conventional, intensitymodulated radiotherapy (B) coplanar (class solution), and (C) non-coplanar plans. 


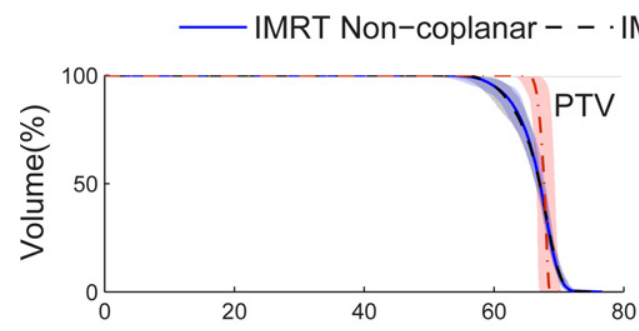

IMRT Coplanar - - - Conventional
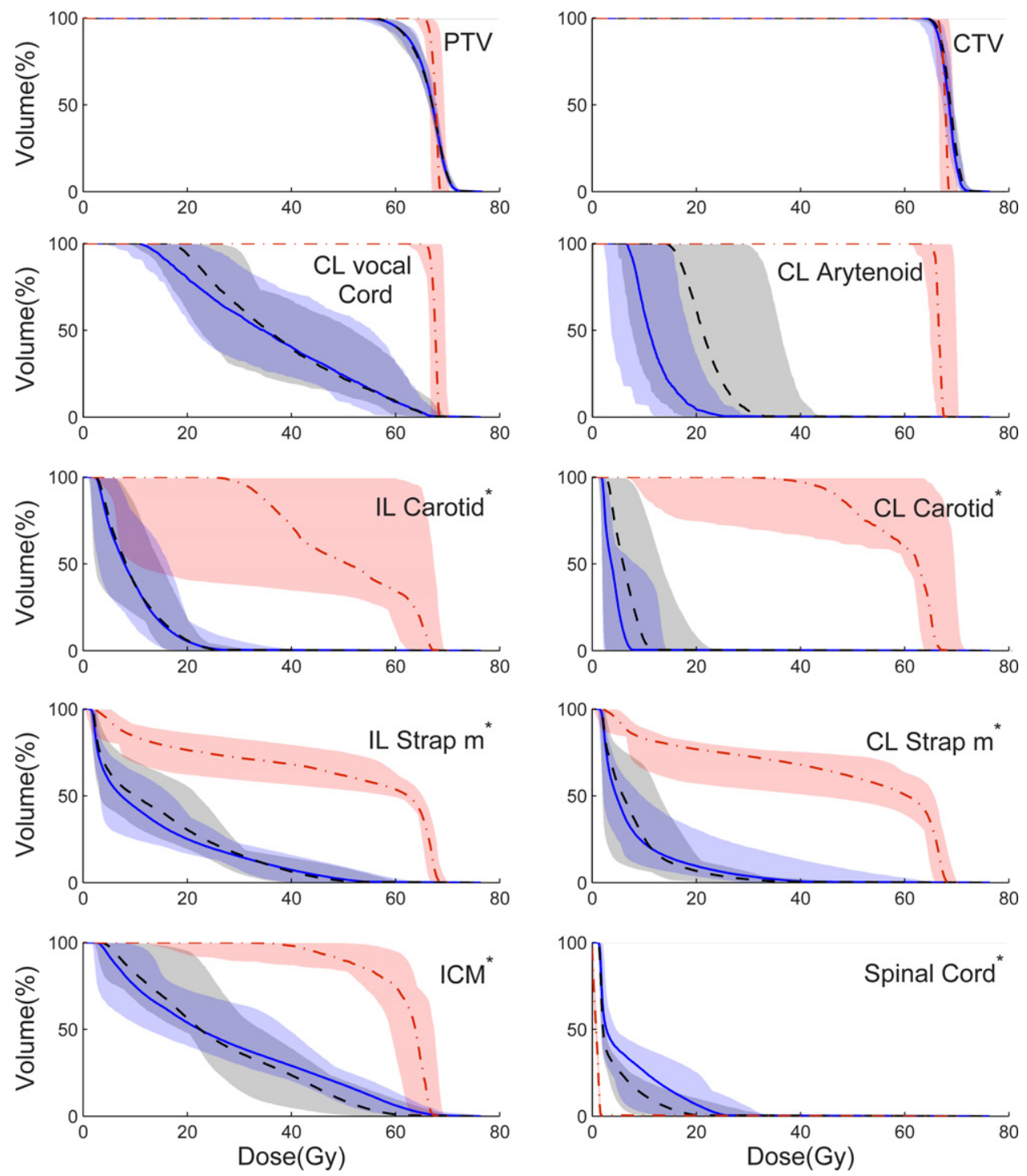

Fig. 6. Population mean dose-volume histograms from different planning techniques shown for selected structures. Shaded areas indicate ranges (population minimal and maximal dose-volume histograms). Asterisk indicates partially contoured organs at risk. $\mathrm{CL}=$ contralateral; $\mathrm{IL}=$ ipsilateral; $\mathrm{m}=$ muscle; $\mathrm{ICM}=$ inferior constrictor muscle.

complications related to RT (e.g., swallowing, speech, persistent edema) $(1,2,23)$. More recent studies have also highlighted the increased risk of strokes and direct carotid artery injury that could result from high-dose irradiation of the carotid arteries (usually $66 \mathrm{~Gy})(3,24)$. Both groups showed a huge reduction of the dose to the carotid arteries when using IMRT for early glottic carcinoma, which was confirmed by our study. Limiting the dose to the carotid arteries might also allow re-irradiation when necessary.

Several groups have opposed the use of IMRT for early glottic cancer $(25,26)$. Their main concern has been that the use of IMRT will compromise local control by failing to deliver adequate doses to a small primary cancer that conventional approaches cannot miss. Another concern has been the limitations of the calculation algorithms that can result in over- or underdosage at the tissue-air interface.
Special attention should also be paid to the potential enhanced occurrence of second primaries with the small fields used in SVCI, because evidence has shown that RT might delay the occurrence of these second tumors (27).

All these valid points were carefully taken into consideration in the present study of SVCI. We have proposed that with the introduction of on-line image-guided RT and the availability of more accurate calculation algorithms in commercially available TPSs, the reduction of the treated volumes is a reasonable step to undertake. In contrast to all previous studies, we defined the CTV (the whole affected vocal cord) contoured on the CT scans with $1-\mathrm{mm}^{3}$ resolution. The intra- and interfractional setup errors have been thoroughly investigated, quantified, and reduced $(6,7)$. Residual geometric errors were used to construct the CTVPTV safety margins. The dose was calculated using MC in 
Table 3. Generalized equivalent uniform dose parameters for different plans

\begin{tabular}{|c|c|c|c|c|c|}
\hline Variable & Conventional plans & IMRT coplanar & $p^{*}$ & IMRT non-coplanar & $p^{\dagger}$ \\
\hline \multicolumn{6}{|l|}{ CTV } \\
\hline $\operatorname{EUD}(-5)$ & $67.6 \pm 1.0$ & $68.6 \pm 0.3$ & $0.03^{\ddagger}$ & $68.3 \pm 0.8$ & 0.3 \\
\hline $\operatorname{EUD}(-20)$ & $67.6 \pm 1.0$ & $68.4 \pm 0.3$ & $0.05^{\ddagger}$ & $68.0 \pm 0.9$ & 0.3 \\
\hline \multicolumn{6}{|l|}{ CL vocal cord } \\
\hline $\operatorname{EUD}(2.2)$ & $67.3 \pm 1.0$ & $41.2 \pm 5.7$ & $0.002^{\ddagger}$ & $40.2 \pm 5.7$ & 0.3 \\
\hline \multicolumn{6}{|l|}{ Ipsilateral arytenoid } \\
\hline $\operatorname{EUD}(2.2)$ & $66.2 \pm 1.4$ & $63.9 \pm 3.9$ & 0.3 & $65.1 \pm 1.7$ & 0.3 \\
\hline \multicolumn{6}{|l|}{ CL arytenoid } \\
\hline EUD(2.2) & $66.2 \pm 1.7$ & $22.1 \pm 8.6$ & $0.002^{\ddagger}$ & $12.6 \pm 4.1$ & $0.01^{\ddagger}$ \\
\hline
\end{tabular}

Abbreviations: $\mathrm{IMRT}=$ intensity-modulated radiotherapy; $\mathrm{CTV}=$ clinical target volume; $\mathrm{EUD}=$ equivalent uniform dose; $\mathrm{CL}=\mathrm{contra}-$ lateral.

* Conventional vs. IMRT coplanar plans.

† Coplanar vs. non-coplanar plans.

¥ Statistically significant.

the Monaco TPS. The results in the present study indicated a tremendous reduction in the doses received by the OARs using the SVCI technique.

In preparation for the SVCI -IMRT technique we have introduced, studies on the dosimetry and dose modeling, especially in the buildup region, must be carefully conducted. In our clinic, some of the IMRT beams from the presented plans were verified using a two-dimensional detector array (15). Gamma analyses showed good agreement between the dose distributions calculated using the Monaco TPS and the measured distributions. Before the clinical introduction of this treatment, more extensive studies on the accuracy of the dose engine near tissue inhomogeneities will be conducted. SVCI should be considered an experimental technique that has yet to be clinically proven. We believe that SVCI should not be applied outside well-controlled clinical studies to prove its efficacy. The extremely high-treatment precision required can only be guaranteed with daily on-line image-guided RT protocols, advanced dose- calculation engines, and a very extensive quality assurance program conducted by experienced personnel.

\section{CONCLUSION}

Evidence was found that image-guided SVCI using IMRT can provide significant sparing of critical structures without compromising CTV coverage, thus offering the possibility of better voice and swallowing function preservation. A huge reduction in the maximal dose received by the carotid arteries was also observed. This has the potential to reduce the risk of stroke and might also allow re-irradiation (if the need arises because of second primaries in the vicinity), especially in younger patients. A class solution of beam angles was presented to ease and standardize treatment planning for a single vocal cord. The proposed technique also offers the new possibilities of dose escalation and hypofractionation for this group of patients.

Table 4. Dosimetric parameters for CTV and contralateral vocal cord for different planning techniques

\begin{tabular}{|c|c|c|c|c|c|}
\hline Variable & Conventional plans & IMRT coplanar & $p^{*}$ & $\begin{array}{c}\text { IMRT } \\
\text { non-coplanar }\end{array}$ & $p^{\dagger}$ \\
\hline \multicolumn{6}{|l|}{ CTV } \\
\hline Mean \pm SD & $100.0 \pm 0.0$ & $99.9 \pm 0.2$ & & $99.6 \pm 0.9$ & \\
\hline Range & 100-100 & 99.4-100.0 & & $97.4-100.0$ & \\
\hline Median & 100.0 & 100.0 & & 100.0 & \\
\hline Mean $\pm \mathrm{SD}$ & $100.0 \pm 0.0$ & $8.1 \pm 6.5$ & & $8.5 \pm 6.7$ & \\
\hline Range & $100.0-100.0$ & $2.0-19.4$ & & $3.0-20.2$ & \\
\hline Median & 100.0 & 5.8 & & 6.3 & \\
\hline $\mathrm{V}_{40 \mathrm{~Gy}}$ & & & $0.002^{\ddagger}$ & & 0.5 \\
\hline Mean \pm SD & $100.0 \pm 0.0$ & $39.9 \pm 14.5$ & & $38.0 \pm 15.3$ & \\
\hline Range & $100.0-100.0$ & $19.2-65.4$ & & $22.0-72.4$ & \\
\hline
\end{tabular}

Abbreviations: $\mathrm{V}_{95 \%}=$ fractional volume that received $\geq 95 \%$ of prescription dose; $\mathrm{SD}=$ standard deviation; $\mathrm{V}_{40 \mathrm{~Gy}}=$ volume receiving dose $\geq 40$ Gy.

* Conventional vs. IMRT coplanar plans.

${ }^{\dagger}$ Coplanar vs. non-coplanar plans.

¥ Statistically significant. 


\section{REFERENCES}

1. Dornfeld K, Simmons JR, Karnell L, et al. Radiation doses to structures within and adjacent to the larynx are correlated with long-term diet- and speech-related quality of life. Int $J$ Radiat Oncol Biol Phys 2007;68:750-757.

2. Rancati T, Schwarz M, Allen AM, et al. Radiation dose-volume effects in the larynx and pharynx. Int J Radiat Oncol Biol Phys 2010;76:S64-S69.

3. Rosenthal DI, Fuller CD, Barker JL Jr., et al. Simple carotidsparing intensity-modulated radiotherapy technique and preliminary experience for T1-2 glottic cancer. Int J Radiat Oncol Biol Phys 2010;77. 455-446.

4. Narayana A, Vaughan AT, Fisher S, et al. Second primary tumors in laryngeal cancer: Results of long-term follow-up. Int J Radiat Oncol Biol Phys 1998;42:557-562.

5. Sewnaik A, Meeuwis CA, van der Kwast TH, et al. Partial laryngectomy for recurrent glottic carcinoma after radiotherapy. Head Neck 2005;27:101-107.

6. Osman SO, de Boer HC, Heijmen BJ, et al. Four-dimensional CT analysis of vocal cords mobility for highly focused single vocal cord irradiation. Radiother Oncol 2008;89:19-27.

7. Osman SO, de Boer HC, Astreinidou E, et al. On-line cone beam CT image guidance for vocal cord tumor targeting. $R a-$ diother Oncol 2009;93:8-13.

8. van Herk M, Remeijer P, Rasch C, et al. The probability of correct target dosage: Dose-population histograms for deriving treatment margins in radiotherapy. Int J Radiat Oncol Biol Phys 2000;47:1121-1135.

9. Hoogeman MS, Nuyttens JJ, Levendag PC, et al. Time dependence of intrafraction patient motion assessed by repeat stereoscopic imaging. Int J Radiat Oncol Biol Phys 2008;70: 609-618.

10. Engelsman M, Rosenthal SJ, Michaud SL, et al. Intra- and interfractional patient motion for a variety of immobilization devices. Med Phys 2005;32:3468-3474.

11. Kian Ang K, Garden AS. Radiotherapy for head and neck cancers: Indications and techniques. 3rd ed. Philadelphia: Lippincott Williams \& Wilkins; 2006.

12. Breedveld S, Storchi PRM, Heijmen BJM. Multi-criteria beam angle IMRT optimization with iCycle. Radiother Oncol 2009; 92:S204.

13. Grofsmid D, Dirkx M, Marijnissen H, et al. Dosimetric validation of a commercial Monte Carlo based IMRT planning system. Med Phys 2010;37:540-549.
14. Jelen $U$, Alber M. A finite size pencil beam algorithm for IMRT dose optimization: Density corrections. Phys Med Biol 2007; 52:617-633.

15. Semenenko VA, Reitz B, Day E, et al. Evaluation of a commercial biologically based IMRT treatment planning system. Med Phys 2008;35:5851-5860.

16. Fippel M. Fast Monte Carlo dose calculation for photon beams based on the VMC electron algorithm. Med Phys 1999;26: 1466-1475.

17. Niemierko A. Reporting and analyzing dose distributions: A concept of equivalent uniform dose. Med Phys 1997;24:103-110.

18. Sanguineti G, Adapala P, Endres EJ, et al. Dosimetric predictors of laryngeal edema. Int J Radiat Oncol Biol Phys 2007; 68:741-749.

19. Meedt G, Alber M, Nüsslin F. Non-coplanar beam direction optimization for intensity-modulated radiotherapy. Phys Med Biol 2003;48:2999-3019.

20. Aref A, Dworkin J, Devi S, et al. Objective evaluation of the quality of voice following radiation therapy for T1 glottic cancer. Radiother Oncol 1997;45:149-153.

21. de Leeuw IMV, Keus RB, Hilgers FJM, et al. Consequences of voice impairment in daily life for patients following radiotherapy for early glottic cancer: Voice quality, vocal function, and vocal performance. Int J Radiat Oncol Biol Phys 1999;44: 1071-1078.

22. Wedman J, Heimdal JH, Elstad I, et al. Voice results in patients with T1a glottic cancer treated by radiotherapy or endoscopic measures. Eur Arch Otorhinolaryngol 2002;259:547-550.

23. Allal AS, Miralbell R, Lehmann W, et al. Effect of arytenoid sparing during radiation therapy of early stage glottic carcinoma. Radiother Oncol 1997;43:63-65.

24. Chera BS, Amdur RJ, Morris CG, et al. Carotid-sparing intensity-modulated radiotherapy for early-stage squamous cell carcinoma of the true vocal cord. Int J Radiat Oncol Biol Phys 2010;77:1380-1385.

25. Mendenhall WM, Amdur RJ, Palta JR. Intensity-modulated radiotherapy in the standard management of head and neck cancer: Promises and pitfalls. J Clin Oncol 2006;24:2618-2623.

26. Feigenberg SJ, Lango M, Nicolaou N, et al. Intensity-modulated radiotherapy for early larynx cancer: Is there a role? Int J Radiat Oncol Biol Phys 2007;68:2-3.

27. Rennemo E, Zätterström U, Evensen J, et al. Reduced risk of head and neck second primary tumors after radiotherapy. $R a$ diother Oncol 2009;93:559-562. 